\title{
The 2nd edition of consensus statements for the diagnosis and management of intestinal Behçet's disease: indication of anti-TNF $\alpha$ monoclonal antibodies
}

\author{
Tadakazu Hisamatsu • Fumiaki Ueno - Takayuki Matsumoto - Kiyonori Kobayashi • \\ Kazutaka Koganei • Reiko Kunisaki - Fumihito Hirai - Masakazu Nagahori • \\ Mitsunobu Matsushita $\cdot$ Kenji Kobayashi $\cdot$ Mitsumasa Kishimoto $\cdot$ \\ Mitsuhiro Takeno $\cdot$ Masanori Tanaka $\cdot$ Nagamu Inoue $\cdot$ Toshifumi Hibi
}

Received: 4 July 2013/Accepted: 6 August 2013/Published online: 18 August 2013

(C) The Author(s) 2013. This article is published with open access at Springerlink.com

\begin{abstract}
Background Clinical evidence regarding intestinal Behçet's disease (BD) management is lacking and intestinal lesions are a poor prognostic factor. In 2007, the Japan consensus statement for diagnosis and management of intestinal BD was developed. Recently, the efficacy of antitumor necrosis factor (TNF) $\alpha$ monoclonal antibodies (mAbs), and infliximab (IFX) was reported and adalimumab (ADA) was approved for intestinal BD in Japan. This study renewed consensus-based practice guidelines for diagnosis and treatment of intestinal BD focusing on the indication of anti-TNF $\alpha$ mAbs.

Methods An expert panel of Japanese gastroenterology and rheumatology specialists was involved. Clinical statements for ratings were extracted from the literature, a professional group survey, and by an expert panel
\end{abstract}

T. Hisamatsu $(\bowtie) \cdot$ T. Hibi

Division of Gastroenterology and Hepatology, Department

of Internal Medicine, School of Medicine, Keio University,

35 Shinanomachi, Shinjuku-ku, Tokyo 160-8582, Japan

e-mail: hisamachi@a7.keio.jp

F. Ueno $\cdot$ K. Kobayashi

Center for Digestive and Liver Diseases, Ohfuna Chuo Hospital,

Kamakura, Japan

T. Matsumoto

Department of Medicine and Clinical Science, Graduate School

of Medical Sciences, Kyushu University, Fukuoka, Japan

K. Kobayashi

Department of Gastroenterology, Kitasato University East

Hospital, Sagamihara, Japan

K. Koganei

Department of Surgery, Yokohama Municipal Citizen's

Hospital, Yokohama, Japan discussion, which rated clinical statements on a nine-point scale. After the first round of ratings, a panelist meeting discussed areas of disagreement and clarified areas of uncertainty. The list of clinical statements was revised after the panelist meeting and a second round of ratings was conducted.

Results Fifteen relevant articles were selected. Based on the first edition consensus statement, improved clinical statements regarding indications for anti-TNF $\alpha$ mAbs use were developed. After a two-round modified Delphi approach, the second edition of consensus statements was finalized.

Conclusions In addition to standard therapies in the first edition, anti-TNF $\alpha$ mAbs (ADA and IFX) should be considered as a standard therapy for intestinal BD. Colchicines, thalidomide, other pharmacological therapy,

\section{R. Kunisaki}

Inflammatory Bowel Disease Center, Yokohama City University

Medical Center, Yokohama, Japan

F. Hirai

Department of Gastroenterology, Fukuoka University Chikushi

Hospital, Chikushino, Japan

M. Nagahori

Department of Gastroenterology and Hepatology, Tokyo

Medical and Dental University, Tokyo, Japan

M. Matsushita

Third Department of Internal Medicine, Kansai Medical

University, Osaka, Japan

M. Kishimoto

Division of Allergy and Rheumatology, St. Luke's International Hospital, Tokyo, Japan 
endoscopic therapy, and leukocytapheresis were deemed experimental therapies.

Keywords Intestinal Behçet's disease · Anti-TNF $\alpha$ $\mathrm{mAb} \cdot$ Consensus statements

$\begin{array}{ll}\text { Abbreviations } \\ \text { ADA } & \text { Adalimumab } \\ \text { BD } & \text { Behçet's disease } \\ \text { CRP } & \text { C-reactive protein } \\ \text { IFX } & \text { Infliximab } \\ \text { mAb } & \text { Monoclonal antibody } \\ \text { TNF } & \text { Tumor necrosis factor }\end{array}$

\section{Introduction}

Behçet's disease (BD) is a chronic relapsing disease with multiple organ system involvement characterized clinically by oral and genital aphthae, cutaneous lesions, and ophthalmological, neurological, or gastrointestinal manifestations [1,2]. Approximately $3-16 \%$ of patients with BD have gastrointestinal tract involvement. Gastrointestinal disease typically affects the ileocecal area, although involvement of the esophagus and small intestine has been reported [3]. The most common gastrointestinal symptoms are abdominal pain, diarrhea, and bleeding. Deep ulcers are responsible for the most common intestinal complications, such as severe bleeding and perforation [4]. Various drugs, such as 5-aminosalicylic acid (5-ASA), systemic corticosteroids, and immunosuppressive agents have been used anecdotally to treat intestinal BD. However, the clinical evidence regarding the management of intestinal $\mathrm{BD}$ is very limited. In 2007, the Japanese Inflammatory Bowel Disease Research Group, supported by the Japanese Ministry of Health, Labour and Welfare, proposed consensus statements for the management of intestinal BD for the first time [5]. In this consensus, infliximab (IFX) was described

\footnotetext{
M. Takeno

Department of Internal Medicine and Clinical Immunology, Yokohama City University Graduate School of Medicine, Yokohama, Japan

M. Tanaka

Department of Pathology and Laboratory Medicine, Hirosaki City Hospital, Hirosaki, Japan

N. Inoue

Center for Preventive Medicine, School of Medicine, Keio University, Tokyo, Japan

T. Hibi

Center for Advanced IBD Research and Treatment, Kitasato University Kitasato Institute Hospital, Tokyo, Japan
}

as an optional therapy for intestinal BD. In recent years, accumulating evidence on the efficacy of anti-TNF $\alpha$ agents for the management of Crohn's disease and Behçet's uveitis have encouraged the use of anti-TNF $\alpha$ agents for management of intestinal BD. Although clinical studies with high-quality evidence have not been available, several cases of intestinal BD successfully treated by anti-TNFa agents have been reported [6-14]. These case reports mainly showed clinical efficacy in the short term, although some reports showed mid- and long-term efficacy and improved endoscopic findings $[15,16]$. Furthermore, on May 16 2013, adalimumab (ADA) was approved as a therapeutic option for intestinal BD in Japan. Currently, the Research Committee for small bowel inflammation of unknown etiology operated by the Health Labour Sciences Research Grant, titled "Research on Measures for Intractable Diseases", was concerned that the approval of anti$\mathrm{TNF} \alpha \mathrm{mAb}$ could dramatically change the therapeutic strategy for intestinal BD. Furthermore, the first edition does not contain information regarding anti-TNF $\alpha$ mAbs and is, therefore, outdated. Therefore, consensus statements for the management of intestinal BD should be adjusted to the current clinical settings, especially regarding the indication of anti-TNF $\alpha$ agents (Table 1).

\section{Methods}

An overview of the study

The development of the second edition of consensus statements for the diagnosis and management of intestinal BD consisted of three phases. In brief, in the first phase, literature that reported the efficacy of anti-TNF $\alpha$ monoclonal antibodies (mAbs) in intestinal BD were collected by survey using PubMed with the following key words: "intestine", "Behçet's disease", "anti-TNF", "infliximab" and "adalimumab". In addition, results of a questionnairebased investigation on the actual treatment situation of intestinal BD by infliximab performed by the Japanese Study Group for a project on Research on Measures for BD operated by the Japanese Ministry of Health, Labour and Welfare in 2012 were referred to. During the second phase, expert panelists discussed areas of disagreement and areas of uncertainty regarding improvements of statements from the first edition and revised some of the clinical statements. During the third phase, the revised clinical statements were rated. Ratings of appropriate methods were developed using a modified Delphi approach, where members of the expert panel rated each part of the statements using a ninepoint scale from 9 to 1 (9, strongly agree; 1 , strongly disagree). Consensus was defined as a median score of $\geq 7$, if the difference between the highest score and lowest score 
Table 1 Consensus statements for the diagnosis and management of intestinal Behçet's disease (second edition), by Research Committee for small bowel inflammation of unknown etiology, and Behçet's Disease Research Committee, Ministry of Health, Labour, and Welfare, Japan

Concept of the second edition of consensus statements

According to increased use of anti-TNF $\alpha$ mAb in inflammatory bowel disease, many cases of intestinal Behçet's disease in which anti-TNF $\alpha$ $\mathrm{mAb}$ (infliximab, IFX) showed efficacy also have been reported in Japan. The same tendency was observed in foreign countries that have a high prevalence of Behçet's disease, such as Korea. In 2013, adalimumab, humanized anti-TNF $\alpha$ mAb was approved for intestinal Behçet's disease in Japan. In the second edition, statements have focused on where we should place anti-TNF $\alpha$ mAb for the treatment of intestinal Behçet's disease based on relevant literature and expert panel discussion. ${ }^{\text {a }}$

\section{Diagnosis}

1. Diagnosis of intestinal Behçet's disease can be made if

A. There is a typical oval-shaped large ulcer in the terminal ileum, OR

B. There are ulcerations or inflammation in the small or large intestine, and clinical findings meet the diagnostic criteria of Behçet's disease. $^{\mathrm{b}}$

2. Acute appendicitis, infectious enteritis, tuberculosis, Crohn's disease, nonspecific colitis, drug-associated colitis and other diseases that mimic intestinal Behçet's disease should be excluded by clinical findings, radiology, and endoscopy before diagnosis of intestinal Behçet's disease is made.

\section{Assessment of severity}

Disease severity should be comprehensively assessed by systemic symptoms (e.g., fever, extra-intestinal manifestations), physical examinations of abdomen (e.g., pain, inflammatory mass, rebound tenderness), depth of ulcers and intestinal complications (e.g., bleeding, stricture, fistula), inflammatory mediators (e.g., CRP, WBC, ESR), and anemia.

\section{Treatment objectives}

In the treatment of intestinal Behçet's disease, as well as the improvement of abdominal and extra-intestinal symptoms, the achievement of negative levels of CRP could be desirable. In the long-term prognosis, the prevention of progression to disability and poly-surgery is important.

A. Standard treatment

1. In patients with severe symptoms (i.e., abdominal pain, diarrhea, gastrointestinal bleeding) and complications with deep ulcers confirmed by radiology or endoscopy, corticosteroids should be considered for induction therapy. The initial dose of corticosteroids is $0.5-1 \mathrm{mg} / \mathrm{kg}$ per day of prednisolone for 1-2 weeks. When clinical improvement is observed, prednisolone should be tapered by $5 \mathrm{mg}$ every week and finally stopped. ADA (approved on May 16, $2013 \mathrm{in} \mathrm{Japan)} \mathrm{could} \mathrm{be} \mathrm{considered} \mathrm{for} \mathrm{induction} \mathrm{therapy} \mathrm{[160} \mathrm{mg} \mathrm{at} 0 \mathrm{w}, 80 \mathrm{mg}$ at $2 \mathrm{w}, 40 \mathrm{mg}$ at $4 \mathrm{w}$, sub-cutaneously (s.c.)]. In responders, scheduled maintenance therapy should be considered (40 mg s.c. every other week). IFX (not approved yet) could also be considered for induction therapy $(5 \mathrm{mg} / \mathrm{kg}$ at week 0,2 , and 6$)$. In responders, scheduled maintenance therapy every 8 weeks should be considered. In patients with mild to moderate activity, mesalasine (5-ASA) could be effective for induction therapy. In patients treated with corticosteroids, anti-TNF $\alpha$ mAbs and immunomodulators, infectious disease and neoplasm should be surveyed. After initiation of these therapies, the risk of infectious disease and neoplasm should be monitored continuously.

2. In patients who are induced to clinical remission, 5-ASA and colchicine could be used for maintenance therapy. The optimal dose of 5-ASA for adult patients is 2.25-3 g/day. When sulfasalazine (SASP) is used, the optimal dose is 3-4 g/day.

3. Immunosuppressive agents such as azathioprine $(\mathrm{AZA})^{\mathrm{c}}$ are indicated when patients are corticosteroid-dependent, corticosteroid-resistant, or anti-TNF $\alpha$ mAb-resistant. The initial dose of AZA is $25-50 \mathrm{mg} / \mathrm{day}$. In patients treated with AZA, adverse effects (e.g., neutropenia and liver dysfunction) should be monitored.

4. Total parenteral nutrition (TPN) is indicated for patients with severe systemic symptoms such as fever and for patients with intestinal complications such as stenosis, fistula, bleeding, and impending perforation. TPN is also indicated for patients who cannot orally intake drugs due to severe oral or upper gastro intestinal lesions. It is usually used for a limited period of time considering the risk of catheter infection and thrombosis. After the patient's condition is improved by TPN, enteral nutrition (EN) could be considered.

5. EN using an elementary diet could be effective for induction therapy. It is indicated in particular for patients with refractory disease, severe activity, and disability such as stricture lesions. When EN is introduced, adherence and quality of life of the patients should be considered.

6. Surgery is indicated for patients in whom improvement is not expected by medications. Patients with severe stricture lesions, perforations, large abscesses, and massive gastrointestinal bleedings have an absolute indication. Patients refractory to medications, and with a low quality of life due to intestinal complications such as fistula, have a relative indication of surgery. Minimum length of resection surgery should be considered.

7. Risk of post-operative recurrence is high in patients with volcano shape deep ulcers and fistulas. Post-operative recurrence often occurs at anastomosis. Although a treatment strategy has not been established that can reduce the risk of post-operative recurrence, considering the high risk of post-operative recurrence and poly surgeries, medication by 5-ASA, immunomodulators, metronidazole, anti-TNF $\alpha$ mAb and EN could be considered for post-operative management.

8. In patients with intestinal Behçet's disease complicated with eye lesions, consultation with ophthalmologists is necessary for their management

B. Optional treatment

- Since there are some case reports showing that spraying of absolute ethanol via endoscope has efficacy for ulcers of intestinal Behçet's, it could be considered in refractory patients. 
Table 1 continued

- Expecting the efficacy as an anti-rheumatoid arthritis drug, change from 5-ASA to SASP could be considered in patients with arthritis (especially peripheral arthritis).

The authors state that, (1) most of the consensus statements are based on expert opinions, (2) the consensus statements have not been endorsed by any organizations, (3) the consensus statements need to be prospectively reevaluated, (4) the consensus statements do not cover histopathological diagnosis, and (5) the consensus statements do not have any binding force.

a The majority of literature regarding anti-TNF $\alpha$ therapy in intestinal Behçet's disease that is referred to for establishment of the second edition described the efficacy of infliximab. On May 16 2013, ADA was approved for intestinal Behçet's disease. The clinical trial of infliximab in intestinal Behçet's disease is currently in progress in Japan.

b Diagnosis of Behçet's disease is according to the Japanese criteria proposed in 2003.

c Immunomodulators besides AZA, including 6-mercaptopurine, cyclosporine, tacrolimus and methotrexate could be considered, but consultations with specialists who have sufficient experience are required. When considering the use of these drugs, adverse effects should be monitored.

was $<4$. For the present study, an expert panel composed of gastroenterologists $(n=6)$, gastrointestinal surgeons $(n=2)$, and rheumatologists $(n=2)$ was established. In addition to the expert panel, a moderator (Hisamatsu, T.) and a professional adviser (Ueno, F.) were involved in the study. The moderator organized discussion by the expert panel and moderated the modified Delphi approach. The moderator searched and reviewed the literature and collected clinical statements. The professional adviser surveyed the process of the modified Delphi approach. The second edition of consensus statements proposed by the expert panel was discussed and then recognized by the Research Committee for small bowel inflammation of unknown etiology operated by a Health Labour Sciences Research Grant, Research on Measures for Intractable Diseases, Japan.

\section{Results}

Search for literature on intestinal BD and anti-TNF $\alpha$ mAbs

In the first phase, 15 relevant literature items were collected. This literature included 10 case reports, 3 retrospective analyses of more than one patient in a single institute, 1 letter to the editor, and 1 review article ("Appendix"). To date, no randomized controlled trials of anti-TNF $\alpha$ mAbs for the treatment of intestinal BD have been reported.

Development of the second edition of consensus statement

In the second phase, the expert panel discussed the place of anti-TNF $\alpha \mathrm{mAb}$ for the treatment of intestinal BD. Based on the literature found, the clinical experience of experts and results of a questionnaire-based investigation, the expert panel agreed that anti-TNF $\alpha \mathrm{mAb}$ treatment should be regarded as a standard therapy for intestinal $\mathrm{BD}$, which was an optional treatment in the first edition. With the recognition of anti-TNF $\alpha \mathrm{mAb}$ treatment as a standard therapy, the expert panel also discussed the therapeutic goal of intestinal BD. In the second edition, it was proposed that the achievement of negative levels of C-reactive protein (CRP) levels, in addition to the improvement of clinical symptoms, could be desirable as an objective therapeutic goal. The expert panel also proposed that improvement of long-term prognosis such as reducing the risk of surgery should be set as a final goal in the treatment of intestinal BD. Corticosteroid and anti-TNF $\alpha$ mAb were placed as standard therapies, while the expert panel deemed colchicines, thalidomide, endoscopic therapy, and leukocytapheresis to be experimental therapies.

In the first round of the modified Delphi approach, there were no statements with a median score $<7$. Although median scores were $\geq 7$, three parts of statements did not obtain consensus because the difference between the highest and lowest score was 4 . After discussion by the expert panel, the second round was performed, and then consensus was obtained for all statements. Thus, after a two-round modified Delphi approach, the second edition of consensus statements was finalized.

The authors' stated that limitations of the second edition included (1) most of the consensus statements are based on expert opinions, (2) the consensus statements have not been endorsed by any organizations, (3) the consensus statements need to be prospectively reevaluated, (4) the consensus statements do not cover histopathological diagnosis, and, (5) the consensus statements do not have any binding force.

\section{Discussion}

BD involves multiple organs, including the eye, nervous system, skin, genitalia, and gastrointestinal tract. About 
$3-16 \%$ of patients with $\mathrm{BD}$ have gastrointestinal tract involvement [3], while most clinical studies of BD published to date concern the management of mucocutaneous lesions and ophthalmological lesions. However, intestinal BD often causes severe gastrointestinal complications, such as massive bleeding and perforation; therefore, intestinal lesions should be considered a poor prognostic factor. Even in high-prevalence areas such as Japan, Korea, the Middle East, and the Mediterranean region, intestinal $\mathrm{BD}$ has been treated empirically because data from the literature regarding management of this condition are scant. The consensus of expert opinion in a high-prevalence area should, therefore, be extremely helpful in daily practice. With this background, the first edition of a consensus for the management of intestinal BD was proposed for the first time in 2007 [5]. However, even after its proposal, conventional therapies have been insufficient for the management of intestinal BD. In the current clinical setting, anti-TNF $\alpha$ mAbs have been used to treat patients with intestinal $\mathrm{BD}$. Reports demonstrating the efficacy of anti-TNF $\alpha \mathrm{mAbs}$ for the management of intestinal BD are increasing. Furthermore, ADA was approved for intestinal BD in 2013 after an open-label clinical trial in Japan. With this in mind, it was considered that the first edition of the consensus statement should be updated.

The first edition was established in 2007 by the Japanese Inflammatory Bowel Disease Research Group. In 2011, the Research Committee for small bowel inflammation of unknown etiology was established independently from the Japanese Inflammatory Bowel Disease Research Group. To avoid changes in expert panel members affecting the results, some members of the first edition joined the expert panel of the second edition, which also had discussions with the Behçet's Disease Research Committee as well as the first edition expert panel. Finally, the second edition was evaluated and approved by the Research Committee for small bowel inflammation of unknown etiology composed of experts for gastrointestinal disorders including members of the first edition.

The modified Delphi approach used in the second edition also provided panelists with the opportunity to discuss their judgments between the rating rounds as well as in the first edition. Unfortunately, there is not much evidence for the management of intestinal BD. Therefore, the discussion by the expert panel must make practical consensus statements rather than be a simple rating method. In the process for improving the second edition of the consensus statement, several subjects were discussed. First, the expert panel discussed the validity of the efficacy of anti-TNF $\alpha$ mAb therapy in intestinal BD. To date, no clinical trial for anti-TNF $\alpha$ mAb therapy in intestinal BD with high-quality evidence such as a double-blind, randomized, placebo-controlled trial has been reported. Therefore, the expert panel relied on their clinical experience and clinical case reports. All members agreed that anti-TNF $\alpha$ mAb therapy is effective for intestinal BD. Second, the expert panel discussed where anti-TNF $\alpha$ mAb therapy should be placed in the treatment of intestinal BD. Although anti-TNF $\alpha$ mAb therapy was considered an option therapy in the first edition in 2007 [5], the expert panel recommended anti-TNF $\alpha \mathrm{mAb}$ as a standard therapy in the second edition. Third, according to the recommendation of anti-TNF $\alpha \mathrm{mAb}$ as a standard therapy, the expert panel discussed whether the goals for medication of intestinal BD should be addressed. The expert panel was concerned about the overuse of anti$\mathrm{TNF} \alpha \mathrm{mAb}$ without any objective parameters. Unfortunately, practical clinical activity indexes for intestinal BD (e.g., Crohn's disease activity index for Crohn's disease) have not been established. Endoscopic mucosal healing was also discussed, but it was not agreed on because of the lack of evidence in the literature and an impractical setting. Although evidence that CRP is a practical biomarker to assess disease activity of intestinal BD is insufficient, several reports suggested that CRP could reflect disease activity and disease prognosis [17]. In addition, in Crohn's disease, negative CRP levels are considered a therapeutic goal as well as endoscopic mucosal healing by biologics therapy. In this context, the expert panel proposed "treatment objectives" that were not in the first edition and recommended the monitoring of CRP.

The problems that now confront us are the safety monitoring of anti-TNF $\alpha$ mAb use and the determination of whether anti-TNF $\alpha \mathrm{mAb}$ treatment can improve the long-term prognosis of intestinal $\mathrm{BD}$ by prospective observation.

\section{Conclusions}

The second edition of consensus statements for the diagnosis and management of intestinal BD was established. In the second edition, anti-TNF $\alpha$ mAb treatment was recognized and recommended as a standard therapy for the treatment of intestinal BD.

Acknowledgments This work was supported in part by grants from the project of Research Committee for small bowel inflammation of unknown etiology and Behçet's Disease Research Committee, Health Labour Sciences Research Grant, Research on Measures for Intractable Diseases, Japan.

Conflict of interest Tadakazu Hisamatsu received a research grant from Ajinomoto Pharmaceuticals CO., LTD. and received lecture fees from Abbvie. 
Open Access This article is distributed under the terms of the Creative Commons Attribution Noncommercial License which permits any noncommercial use, distribution, and reproduction in any medium, provided the original author(s) and the source are credited.

\section{Appendix: literature list of intestinal Behçet's disease and anti-TNF $\alpha$ mAbs treatment}

- Travis SP, Czajkowski M, McGovern DP, Watson RG, Bell AL. Treatment of intestinal Behçet's syndrome with chimeric tumour necrosis factor alpha antibody. Gut. 2001;49(5):725-8.

- Hassard PV, Binder SW, Nelson V, Vasiliauskas EA. Anti-tumor necrosis factor monoclonal antibody therapy for gastrointestinal Behçet's disease: a case report. Gastroenterology. 2001;120(4):995-9.

- Kram MT, May LD, Goodman S, Molinas S. Behçet's ileocolitis: successful treatment with tumor necrosis factor-alpha antibody (infliximab) therapy: report of a case. Dis Colon Rectum. 2003;46(1):118-21.

- Pipitone N, Olivieri I, Cantini F, Triolo G, Salvarani C. New approaches in the treatment of AdamantiadesBehçet's disease. Curr Opin Rheumatol. 2006;18(1): 3-9. Review.

- Byeon JS, Choi EK, Heo NY, Hong SC, Myung SJ, Yang SK, Kim JH, Song JK, Yoo B, Yu CS. Antitumor necrosis factor-alpha therapy for early postoperative recurrence of gastrointestinal Behçet's disease: report of a case. Dis Colon Rectum. 2007;50(5):672-6.

- Ju JH, Kwok SK, Seo SH, Yoon CH, Kim HY, Park SH. Successful treatment of life-threatening intestinal ulcer in Behçet's disease with infliximab: rapid healing of Behçet's ulcer with infliximab. Clin Rheumatol. 2007;26(8):1383-5.

- Lee JH, Kim TN, Choi ST, Jang BI, Shin KC, Lee SB, Shim YR. Remission of intestinal Behçet's disease treated with anti-tumor necrosis factor alpha monoclonal antibody (infliximab). Korean $\mathrm{J}$ Intern Med. 2007;22(1):24-7.

- Ugras M, Ertem D, Celikel C, Pehlivanoglu E. Infliximab as an alternative treatment for Behçet disease when other therapies fail. J Pediatr Gastroenterol Nutr. 2008;46(2):212-5.

- Naganuma M, Sakuraba A, Hisamatsu T, Ochiai H, Hasegawa H, Ogata H, Iwao Y, Hibi T. Efficacy of infliximab for induction and maintenance of remission in intestinal Behçet's disease. Inflamm Bowel Dis. 2008;14(9):1259-64.

- Ariyachaipanich A, Berkelhammer C, Nicola H. Intestinal Behçet's disease: maintenance of remission with adalimumab monotherapy. Inflamm Bowel Dis. 2009;15(12):1769-71.
- Iwata S, Saito K, Yamaoka K, Tsujimura S, Nawata M, Suzuki K, Tanaka Y. Effects of anti-TNF-alpha antibody infliximab in refractory entero-Behçet's disease. Rheumatology (Oxford). 2009;48(8):1012-3.

- Kaneko U, Kishi T, Kikuchi M, Hara R, Shinoki T, Miyamae T, Imagawa T, Mori M, Yokota S. Two patients with childhood-onset Behçet's disease successfully treated by anti-tumor necrosis factor therapy. Nihon Rinsho Meneki Gakkai Kaishi. 2010;33(3): 157-61. (In Japanese).

- Donghi D, Mainetti C. Infliximab for the treatment of refractory Adamantiades-Behçet disease with articular, intestinal, cerebral and ocular involvement. Dermatology. 2010;220(3):282-6.

- Iwata S, Saito K, Yamaoka K, Tsujimura S, Nawata M, Hanami K, Tanaka Y. Efficacy of combination therapy of anti-TNF- $\alpha$ antibody infliximab and methotrexate in refractory entero-Behçet's disease. Mod Rheumatol. 2011;21(2):184-91.

- Maruyama Y, Hisamatsu T, Matsuoka K, Naganuma M, Inoue N, Ogata H, Iwao Y, Kanai T, Hibi T. A case of intestinal Behçet's disease treated with infliximab monotherapy who successfully maintained clinical remission and complete mucosal healing for six years. Intern Med. 2012;51(16):2125- 9.

\section{References}

1. Garton RA, Ghate JV, Jorizzo JL. Behçet's disease. In: Harris Jr ED, Budd RC, Genovese MC, Firestein GS, Sargent JS, Sledge $\mathrm{CB}$, editors. Textbook of rheumatology. 7th ed. Philadelphia: Saunders; 2005.

2. Krause I, Weinberger A. Behçet's disease. Curr Opin Rheumatol. 2008;20(1):82-7 (Review).

3. Sakane T, Takeno M, Suzuki N, Inaba G. Current concepts: Behçet disease. New Eng J Med. 1999;341:1284-91.

4. Brandt LJ, Boley SJ. Intestinal ischemia. In: Feldman M, Friedman LS, Sleisenger MH, editors. Gastrointestinal and liver disease. 7th ed. Philadelphia: Saunders; 2002.

5. Kobayashi K, Ueno F, Bito S, Iwao Y, Fukushima T, Hiwatashi N, Igarashi M, Iizuka BE, Matsuda T, Matsui T, Matsumoto T, Sugita A, Takeno M, Hibi T. Development of consensus statements for the diagnosis and management of intestinal Behçet's disease using a modified Delphi approach. J Gastroenterol. 2007;42(9):737-45.

6. Travis SP, Czajkowski M, McGovern DP, Watson RG, Bell AL. Treatment of intestinal Behçet's syndrome with chimeric tumour necrosis factor alpha antibody. Gut. 2001;49(5):725-8.

7. Hassard PV, Binder SW, Nelson V, Vasiliauskas EA. Anti-tumor necrosis factor monoclonal antibody therapy for gastrointestinal Behçet's disease: a case report. Gastroenterology. 2001;120(4): 995-9.

8. Kram MT, May LD, Goodman S, Molinas S. Behçet's ileocolitis: successful treatment with tumor necrosis factor-alpha antibody (infliximab) therapy: report of a case. Dis Colon Rectum. 2003;46(1):118-21.

9. Byeon JS, Choi EK, Heo NY, Hong SC, Myung SJ, Yang SK, Kim JH, Song JK, Yoo B, Yu CS. Antitumor necrosis factor- 
alpha therapy for early postoperative recurrence of gastrointestinal Behçet's disease: report of a case. Dis Colon Rectum. 2007;50(5):672-6.

10. Lee JH, Kim TN, Choi ST, Jang BI, Shin KC, Lee SB, Shim YR. Remission of intestinal Behçet's disease treated with anti-tumor necrosis factor alpha monoclonal antibody (infliximab). Korean J Intern Med. 2007;22(1):24-7.

11. Ju JH, Kwok SK, Seo SH, Yoon CH, Kim HY, Park SH. Successful treatment of life-threatening intestinal ulcer in Behçet's disease with infliximab: rapid healing of Behçet's ulcer with infliximab. Clin Rheumatol. 2007;26(8):1383-5.

12. Ugras M, Ertem D, Celikel C, Pehlivanoglu E. Infliximab as an alternative treatment for Behçet disease when other therapies fail. J Pediatr Gastroenterol Nutr. 2008;46(2):212-5.

13. Naganuma M, Sakuraba A, Hisamatsu T, Ochiai H, Hasegawa $H$, Ogata H, Iwao Y, Hibi T. Efficacy of infliximab for induction and maintenance of remission in intestinal Behçet's disease. Inflamm Bowel Dis. 2008;14(9):1259-64.
14. Donghi D, Mainetti C. Infliximab for the treatment of refractory Adamantiades-Behçet disease with articular, intestinal, cerebral and ocular involvement. Dermatology. 2010;220(3):282-6.

15. Iwata S, Saito K, Yamaoka K, Tsujimura S, Nawata M, Hanami $\mathrm{K}$, Tanaka Y. Efficacy of combination therapy of anti-TNF- $\alpha$ antibody infliximab and methotrexate in refractory entero-Behçet's disease. Mod Rheumatol. 2011;21(2):184-91.

16. Maruyama $Y$, Hisamatsu T, Matsuoka K, Naganuma M, Inoue N, Ogata H, Iwao Y, Kanai T, Hibi T. A case of intestinal Behçet's disease treated with infliximab monotherapy who successfully maintained clinical remission and complete mucosal healing for six years. Intern Med. 2012;51(16):2125-9.

17. Jung YS, Cheon JH, Park SJ, Hong SP, Kim TI, Kim WH. Clinical course of intestinal Behçet's disease during the first five years. Dig Dis Sci. 2013;58(2):496-503. 